Original research article

\title{
Association between hemostatic markers, serum lipid fractions and progression of cerebral small vessel disease: A 2-year follow-up study
}

\author{
Jacek Staszewski ${ }^{a,}{ }^{*}$, Renata Piusińska-Macoch ${ }^{a}$, Bogdan Brodacki ${ }^{a}$, \\ Ewa Skrobowska ${ }^{b}$, Adam Stępień ${ }^{a}$ \\ ${ }^{a}$ Clinic of Neurology, Military Institute of Medicine, Szaserow 128, Warsaw 04-141, Poland \\ ${ }^{\mathrm{b}}$ Department of Radiology, Military Institute of Medicine, Szaserow 128, Warsaw 04-141, Poland
}

\section{A R T I C L E I N F O}

\section{Article history:}

Received 6 July 2017

Accepted 2 November 2017

Available online 14 November 2017

Keywords:

Cerebral small vessel disease

Radiological progression

Risk factor

Biochemical marker

Fibrinogen

\begin{abstract}
A B S T R A C T
Introduction: Little is known if hemostatic markers and serum lipid fractions can predict further radiological progression beyond vascular risk factors in cerebral small vessel disease (SVD). We investigated whether they are associated with SVD radiological progression and if they are related to different SVD clinical manifestations.

Methods: A single-center, prospective, cohort study with 2 years of radiological follow-up was performed in consecutive patients with different SVD manifestations. The study group consisted of 123 patients: 49 with lacunar stroke (LS), 48 with vascular dementia (VaD) and 26 with vascular parkinsonism (VaP). We assessed SVD progression by a visual SVD scale. We determined the relationship between serum or plasma concentrations of tissue factor (TF), thrombomodulin, beta-thromboglobulin (BTG), fibrinogen, D-dimer and total cholesterol, HDL-C, LDL-C, triglycerides and SVD progression by logistic regression analysis.

Results: $34.9 \%$ patients had SVD radiological progression: $43 \%$ had isolated WMLs progression, $23.2 \%$ had new lacunes, 34.8\% had both WMLs progression and new lacunes. Fibrinogen [OR 1.02 (95\% CI 1.006-1.011] was significantly associated with risk of new lacunes or WMLs progression regardless of the clinical SVD manifestation. While low HDL [OR 0.96 (0.93-1)] and TF [OR 1.07 (0.99-1.1)] were marginally associated with new lacunes, BTG [OR 1.005 (0.991.01)] was associated with WMLs progression.

Conclusion: We found a relationship between fibrinogen and risk of radiological progression of SVD regardless of the clinical SVD manifestation. In addition, lower HDL and increased TF predicted development of new lacunes, and higher BTG was associated with risk of WMLs progression.
\end{abstract}

๑ 2017 Published by Elsevier Sp. z o.o. on behalf of Polish Neurological Society.

\footnotetext{
* Corresponding author at: Clinic of Neurology, Military Institute of Medicine, Szaserow 128, 04-141 Warsaw, Poland.

E-mail address: jstaszewski@wim.mil.pl (J. Staszewski).

https://doi.org/10.1016/j.pjnns.2017.11.005

0028-3843/@ 2017 Published by Elsevier Sp. z o.o. on behalf of Polish Neurological Society.
} 


\section{Introduction}

Cerebral small vessel disease (SVD) is one of the most important and common vascular diseases of the brain caused by lacunar infarcts (LI) and white matter lesions (WMLs). It refers to different but pathogenetically linked processes and mechanisms: atherosclerosis, arteriolosclerosis, lipohyalinosis but also it results from increased permeability of the bloodbrain-barrier (BBB) [1]. Features of SVD probably develop over many years before becoming clinically evident, hence SVD can cause several different types of distinct or overlapping clinical presentations such as progressive cognitive decline or vascular dementia (VaD), physical disabilities e.g. vascular parkinsonism (VaP), but also it often results in sudden onset lacunar stroke (LS) [2]. Recently the number of lesions attributable to SVD has expanded. The main imaging features of SVD are now recognized all to be inter-related and visible on conventional magnetic resonance imaging (MRI). They include acute LIs or hemorrhages, lacunes (which reflect old infarcts), WMLs, visible perivascular spaces (PVS), microbleeds (MCBs), and brain atrophy [2]. Lacunes and WMLs are the hallmarks of SVD, they often coexist, but in some patients one type of imaging appearance may predominate. Whether they share similar or different pathogenesis and risk factors is a matter of debate. Population-based studies revealed that age-related WMLs are present in MRI in over 95\% of elderly individuals aged between 60 and 90 years [3]. The Leukoaraiosis and Disability Study (LADIS) found WMLs progression in 74\% of participants over 3 years particularly in those with confluent lesions at baseline [4]. Progression of SVD is associated with risk of stroke, cognitive decline, gait abnormalities and disability, and therefore it is important to identify associated factors especially those that could be treatable. The relationships of traditional risk factors with SVD are still not completely understood and atherothrombotic risk factors are not consistently common in patients with different SVD manifestations. In a systematic review of 16 studies comparing risk factors between patients with different stroke etiology, hypertension and diabetes were more frequent in patients with LS than in large vessel strokes [5]. Most community-based studies consistently showed that age, hypertension and smoking were associated with WMLs, however they failed to find an association with diabetes [6]. The majority of studies demonstrated that older age and hypertension predicted WMLs progression, however studies on association between diabetes, cholesterol levels or statin use gave conflicting results $[4,7]$.

Although the clinical and radiological course of SVD was evaluated in many studies especially focusing on patients with LS or asymptomatic SVD patients, the prognosis of SVD and predictors of radiological progression, especially in chronic SVD manifestations e.g. VaP or VaD are not well known and have been rarely studied [8]. Variability in clinical and radiological presentations of SVD may be attributable to burden of traditional vascular risk factors leading to endothelial dysfunction, but also may be due to other hemodynamic or hemostatic factors, however their significance in the pathogenesis and prognosis of SVD is not clear. Given the spectrum of SVD pathological pathways - from BBB dysfunction to atherosclerosis it is likely that several components of fibrinolytic or inflammatory-atherothrombotic cascade are involved. Studies investigating association between different biochemical compounds e.g. serum lipid fractions and hemostatic markers and risk of LS or SVD radiological burden gave conflicting results [9]. Previous studies have suggested that the blood viscosity measured in LS was higher than in other stroke subtypes and that increasing triglycerides concentration and decreased LDL cholesterol were associated with WMLs in elderly population $[10,11]$. However, little is known if they can predict further radiological progression in SVD and add predictive capacity beyond vascular risk factors. We hypothesized that specific markers involved in fibrinolytic process and lipid metabolism are linked to risk of SVD radiological progression and examined if they are related to different SVD clinical manifestations: LS, VaD or VaP.

\section{Materials and methods}

A single-center, prospective cohort study, with 2 years of radiological follow-up was performed as a part of SHEF-CSVD Study (Significance of HEmodynamic and hemostatic Factors in the course of different manifestations of Cerebral Small Vessel Disease) [12]. The study group consisted of 132 patients ( 54 with LS, 50 with $\mathrm{VaD}, 28$ with $\mathrm{VaP}$ ) considered to be caused by sporadic SVD of whom 7 patients died and 2 withdrawn a consent during follow-up. Finally 123 patients were analyzed: 49 with LS (39.8\%), 48 with $\operatorname{VaD}(39 \%)$ and 26 with $\operatorname{VaP}(21.1 \%)$ (Fig. 1). Baseline demographic data, clinical and radiological characteristics of patients who were excluded from final analysis were comparable to those of the included patients. The patients were recruited from Neurological Outpatient Department and prospectively enrolled to the study between December 2011 and June 2014. The MRI examination was performed at baseline and repeated at the end of the 2-year follow-up with the aim of assessing possible progression of WMLs and development of new lacunes or other lesions. Given the nature of this study, the aim of research is hypothesisgenerating rather than confirmatory. The study protocol and methods have been thoroughly described elsewhere [12].

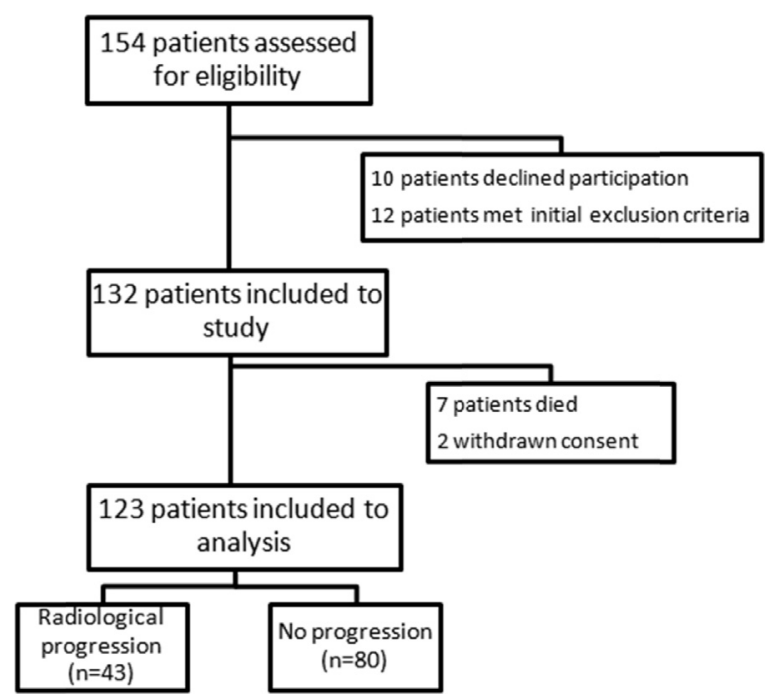

Fig. 1 - A flowchart of study design. 
In brief, SVD group consisted of consecutive patients with a first ever recent LS or newly diagnosed $\mathrm{VaD}$ and $\mathrm{VaP}$ presumed to be caused by sporadic SVD. Although SVD remains a heterogeneous disease, the majority of SVD especially in elderly is sporadic and the core mechanism underlying brain injury is assumed to be ischemia acting through arteriolar narrowing or failure of cerebral autoregulation. However, LS may occasionally be caused by other etiologies (e.g. artery-to-artery or cardiac embolism), and similarly, WMLs may be seen with other vascular or nonvascular conditions (e.g. due to CADASIL, vasculitis, amyloid angiopathy, multiple sclerosis, sarcoidosis or Lyme disease). Every effort was taken to perform the differential diagnosis to include patients with sporadic SVD only. The patients were diagnosed based on the complex evaluation of past medical history, clinical context, available diagnostic tests (e.g. carotid and transcranial duplex ultrasound, holter ECG) and according to typical radiological and clinical picture: LS - according to the OCSP Criteria; VaD and VaP after exclusion of other neurodegenerative conditions with the use of clinical tools easily applied in clinical practice: Hurtig criteria or NINDS-AIREN criteria with Modified Hachinski Ischemic Scale $\geq 7$ points, respectively [13-15]. Patients with non-SVD related WMLs, significant stenosis ( $\geq 50 \%$ ) of a major extracranial or intracranial artery, atrial fibrillation, life expectancy of less than 6 months, and MRI contraindications were excluded. Participants were aged between 60 and 90 years, they were independent (total Barthel Index $\geq 80$ points) and had no severe dementia (MMSE $\geq 12$ points). Those with recurrent LS or strategic single-infarct dementia or with poststroke $\mathrm{VaD}$ or $\mathrm{VaP}$ were not included. Mean time from first symptoms to enrollment was $23.2 \pm 10$ months in VaD and 25 \pm 10 months in $\operatorname{VaP}(p=0.5)$. To prevent confounding by hyperacute phase responses, all LS patients underwent study procedures at least 2 weeks (mean $17.5 \pm 3.8$ days) after their index strokes. The study complied with the Declaration of Helsinki. All participants signed an informed consent form. The study was approved by the local Medical Ethics Committee (46/WIM/2010).

\subsection{Procedure}

\subsubsection{Laboratory examinations}

We investigated a set of 9 markers of hemostasis (tissue factor [TF], thrombomodulin [TM], beta-thromboglobulin [BTG], fibrinogen, D-dimer) and lipid metabolism (serum total cholesterol [TC], high density lipoprotein cholesterol [HDL-C], low-density lipoprotein cholesterol [LDL-C], triglycerides [TG]). Fasting morning samples were collected at baseline into an EDTA containing tube, centrifuged and fractionated at $3000 \times \mathrm{g}$ for $15 \mathrm{~min}$ at $4{ }^{\circ} \mathrm{C}$ and stored at $-80^{\circ} \mathrm{C}$. Tissue factor, TM, BTG measurements were performed using commercially available ELISA kits according to manufacturer protocol (Bio-Source, Europe, Nivelles, Belgium). For each sample, duplicate readings were performed, intra-assay coefficients of variation was $<10 \%$. Other biochemical markers were measured by automatic biochemical analyzer. In order to improve the wording in manuscript, whenever a compound or metabolite is mentioned, we actually mean its serum or plasma concentration.

\subsubsection{MRI evaluation}

MRI examinations were performed at baseline and after 2 years (mean $22.7 \pm 3.7$ months; LS $22.6 \pm 3.8$; VaP $21.7 \pm 4.3$; $\operatorname{VaD} 23.2 \pm 3.3$ months, ANOVA $p=0.28$ ). For MRI evaluation we used imaging with standard T2-weighted, FLAIR and gradient echo sequences. All MRI scans were obtained using the same scanner (GE Healthcare 1.5 T scanner). Baseline and follow-up scans were read independently by the same experienced investigator (E.S.) blinded to the clinical diagnosis and used to calculate change scores over time. We categorized MRI findings according to STRIVE (Standards for Reporting Vascular Changes on Neuroimaging) guidelines as a reference standard [16]. As imaging features of SVD can be quantified they were used as markers of the presence and severity of SVD in the study. Images were rated for the presence of lacunes, WMLs, MCBs, and PVS according to a recommended visual SVD scale which provides a more complete overall view of the impact of SVD on the brain than do the individual MRI features separately [17].

White matter lesions. Periventricular (pWMLs) and deep WMLs (dWMLs) were visualized on T2 and PD/FLAIR images as ill-defined hyperintensities $\geq 5 \mathrm{~mm}$. The simple modified Fazekas rating scale was used to estimate the extent of the pWMLs and dWMLs as this scale demonstrated good interrater agreement and correlated sufficiently with the volume of white matter damage derived from fully quantitative measurements [18-20]. Mild WMLs (grade 1) was defined as punctate lesions in the deep white matter with a maximum diameter of $9 \mathrm{~mm}$ for a single lesion and of $20 \mathrm{~mm}$ for grouped lesions. Moderate WMLs (grade 2) were early confluent lesions of $10-20 \mathrm{~mm}$ single lesions and $>20 \mathrm{~mm}$ grouped lesions in any diameter, and no more than connecting bridges between the individual lesions. Severe WMLs (grade 3) were single lesions or confluent areas of hyperintensity of $\geq 20 \mathrm{~mm}$ in any diameter. All studied patients had at least Fazekas grade 1 WMLs. One point in SVD scale was awarded if (early) confluent deep WMLs (Fazekas score 2 and 3) or irregular periventricular hyperintensities extending into the deep white matter (Fazekas score 3) were present.

Lacunes. One point on the SVD scale was awarded when 1 or more lacunes (small $<15 \mathrm{~mm}$, subcortical lesions of similar signal to CSF, i.e., increased signal on T2-weighted, decreased signal on FLAIR and T1-weighted images) were present.

Cerebral microbleeds. Cerebral microbleeds were defined as small $(<5 \mathrm{~mm})$, homogeneous, round foci of low signal intensity on $\mathrm{T} 2{ }^{*}$-weighted images in basal ganglia, thalamus, white matter, or cortico-subcortical junction. The presence of 1 or more microbleeds gave 1 point on the SVD scale.

Enlarged perivascular spaces. We defined PVS as small $(<3 \mathrm{~mm}$ ) punctate (if perpendicular) and linear (if longitudinal to the plane of scan) hyperintensities on T2 images. One point was awarded if moderate to extensive (10-25 or $>25)$ enlarged perivascular spaces were present [21].

Finally the presence of each of the above markers was awarded with 1 point, producing a minimum score of 0 and a maximum of 4 , representing the total MRI load of SVD.

The complete set of baseline MRI variables of interest, included qualitative assessment of brain LIs, WMLs, MCBs, and cortical atrophy, was compared with those obtained from follow-up MRI scans. 
Assessment of radiological progression. At follow-up, visual rating of SVD radiological progression expressed by WMLs progression or development of new lacunes was performed. WMLs progression and lacunes were rated on FLAIR images as proposed by Prins et al., in which absence or presence of progression ( 0 and 1 , respectively) was rated in 3 periventricular regions (frontal caps, occipital caps, bands), 4 subcortical white matter regions (frontal, parietal, occipital, temporal), basal ganglia, and infratentorial region [22]. We defined radiological SVD progression as an increase of WMLs (WMLs progression group) or new lacunes (new lacunes group) in one or more periventricular and/or subcortical regions.

\subsubsection{Atherothrombotic risk evaluation}

Based on medical records, physical examination and comprehensive history available at baseline, we evaluated atherothrombotic risk factors, including tobacco use, diabetes, hyperlipidemia, hypertension, coronary artery disease (CAD) and peripheral vascular disease (PAD). Hypertension was defined as systolic BP (SBP) $\geq 140 \mathrm{~mm} \mathrm{Hg}$ and/or diastolic $\mathrm{BP} \geq 90 \mathrm{~mm} \mathrm{Hg}$, or use of antihypertensive medication. Diabetes mellitus was defined as fasting serum glucose concentration $\geq 126 \mathrm{mg} / \mathrm{dl}$, hemoglobin A1c $\geq 6.5 \%$, or use of antidiabetic medication. The following criteria were used to diagnose polymetabolic syndrome: waist circumference $\geq 102 \mathrm{~cm}$ in men or $\geq 88 \mathrm{~cm}$ in women; $\mathrm{HDL}<40 \mathrm{mg} / \mathrm{dl}$ in men and $<50 \mathrm{mg} / \mathrm{dl}$ in women or on drug treatment; elevated blood pressure $\geq 130 \mathrm{mmHg}$ systolic or $\geq 85 \mathrm{mmHg}$ diastolic or on drug treatment; elevated $\mathrm{TG} \geq 150 \mathrm{mg} / \mathrm{dl}$ or on drug treatment; and elevated fasting glucose (FG) $\geq 100 \mathrm{mg} / \mathrm{dl}$ or on treatment for diabetes. Coronary artery disease (CAD) was defined in patients with stable angina, prior myocardial infarctions (MIs), prior percutaneous revascularization, coronary artery bypass graft, angiographically proven coronary atherosclerosis, or reliable non-invasive evidence of myocardial ischemia. Previous history of CAD and peripheral artery disease (PAD) was recorded based on clinical history and documented investigations. Patients who were smokers at the time of analysis were classified as current smokers. To determine baseline blood pressure control we performed $24 \mathrm{~h}$ ABPM using a portable non-invasive oscillometric and auscultatory device (Schiller MT-300). All patients received optimal medical treatment, according to guidelines. As we reported previously, there were no significant differences with regard to mean age and distribution of other vascular risk factors between patients with $\mathrm{VaD}$ and $\mathrm{VaP}$ [23].

\subsection{Statistical analysis}

Log-normal data were compared using paired t-tests, nonnormal data were analyzed using nonparametric tests, the chisquare test was used for comparisons of categorical variables. All markers were studied as continuous variables. The difference between baseline risk factors and concentration of biochemical compounds was compared between patients without and with any radiological progression, with WMLs progression or new lacunar lesions only, and between patients with LS, VaD and VaP. All variables that achieved a $p$-value $\leq 0.1$ in any of the analysis were included in the multivariate regression model as candidate variables and then removed by backward stepwise selection procedure. Backward elimination of nonsignificant variables $(p>0.1)$ was subsequently used to generate a minimal model. We assessed the relationship between analyzed factors at baseline (independent factors) and SVD progression at 2-years follow-up (dependent factor). The results are presented as the odds ratio (OR) with $95 \%$ confidence intervals (CI). We performed three separate analyses: (1) any progression of WMLs and/or development of new lacunes, (2) development of new lacunes, (3) WMLs progression. To evaluate a variable's power to predict SVD progression, we also used receiver operating characteristic (ROC). Due to the small sample size threshold-, sensitivity and specificity values were not reported. Statistical significance was set at $p$-value $<0.05$ in all analyses. All data are presented as mean $\pm \mathrm{SD}$ values. All analyses were performed using Statistica 12 software (StatSoft Inc, USA).

\section{Results}

\subsection{Patient characteristics}

Above one-third of patients (34.9\%) experienced radiological progression during the study flow. Isolated WMLs progression was the most common finding and was discovered in $43 \%$ $(n=18)$ of these cases, new lacunes in $23.2 \%(n=10)$, both WMLs progression and new lacunes in 15 patients (34.8\%). Mean age, sex distribution, prevalence of major vascular risk factors did not significantly differ between patients with and without radiological progression, however more than half of patients who experienced progression had VaD (Table 1). Baseline total SVD load on MRI assessed in SVD scale and Fazekas scale were similar in patients without and with radiological progression, however those who progressed had higher load of deep WMLs (Table 2). Also the prevalence of lacunes, CMBs, PVS and brain atrophy at baseline did not differ significantly between groups.

\subsection{Biochemical markers}

There were significant differences between patients with and without radiological progression with regard to concentration of fibrinolysis markers and lipid metabolites: patients who had progressed had significantly elevated fibrinogen, D-dimer, TF and lower TC (Table 3). There were also some differences in biochemical profile between patients with diverse markers of radiological progression. Compared with patients without progression, those with new lacunes had significantly lower HDL and higher TF while patients with new WMLs had lower eGFR and tended to have higher BTG.

Fibrinogen and HDL were the only markers significantly associated with risk of any radiological progression in multivariable analysis. Moreover, TF and BTG were marginally associated with risk of new lacunes and new WMLs, respectively (Table 4). Additional analyses after adjustment for age and sex or baseline Fazekas score did not change these results essentially. We also made a comparison between patients with different clinical manifestations of SVD who experienced radiological progression. Fibrinogen was significantly elevated in all SVD groups. Patients with VaD who 
Table 1 - Baseline characteristics of studied patients without and with radiological progression.

\begin{tabular}{|c|c|c|c|}
\hline Variables & No progression & Radiological progression & $p$ \\
\hline$n$ & 80 & 43 & - \\
\hline \multicolumn{4}{|l|}{ SVD manifestation; $N(\%)$} \\
\hline LS & $37(46.3)$ & $12(27.9)$ & \multirow[t]{3}{*}{0.09} \\
\hline $\mathrm{VaD}$ & $26(32.5)$ & $22(51.2)$ & \\
\hline $\mathrm{VaP}$ & $17(21.3)$ & $9(20.9)$ & \\
\hline Age. mean $( \pm S D)$ years & $71.25(8.07)$ & $73.8(8)$ & 0.1 \\
\hline \multicolumn{4}{|l|}{ Age/decades } \\
\hline$<65$ yrs & $34(42.5)$ & $14(32.6)$ & \multirow[t]{3}{*}{0.47} \\
\hline $65-75$ yrs & $30(37.5)$ & $17(39.5)$ & \\
\hline$>75$ yrs & $16(20)$ & $12(27.9)$ & \\
\hline Female sex & $41(51.3)$ & $19(44.2)$ & 0.57 \\
\hline Hypertension & $69(86.3)$ & $37(86)$ & 0.99 \\
\hline 24h MAP & $93.11(11.7)$ & $97.3(12)$ & 0.15 \\
\hline Mean $24 \mathrm{~h}$ SBP & $132.4(16.9)$ & $135.2(16.7)$ & 0.4 \\
\hline Mean 24 h DBP & $73.4(11)$ & $78.4(11)$ & 0.11 \\
\hline CAD & $18(22.5)$ & $6(14)$ & 0.34 \\
\hline Diabetes mellitus & $46(56.3)$ & $23(53.5)$ & 0.85 \\
\hline Current smoking & $25(31.3)$ & $12(27.9)$ & 0.83 \\
\hline Hyperlipidemia & $62(77.5)$ & $29(67.4)$ & 0.28 \\
\hline PAD & $5(6.3)$ & $5(11.6)$ & 0.31 \\
\hline PS & $37(46.3)$ & $16(37.2)$ & 0.34 \\
\hline Obesity (BMI $\geq 30 \mathrm{~kg} / \mathrm{m}^{2}$ ) & $63(78.8)$ & $29(67.4)$ & 0.19 \\
\hline CKD & $9(11.3)$ & $9(20.9)$ & 0.18 \\
\hline Use of statin medications & $64(80)$ & $32(74.4)$ & 0.49 \\
\hline Use of antihypertensive therapy & $67(83)$ & $35(81)$ & 0.81 \\
\hline Daily use of aspirin & $68(85)$ & $32(74.4)$ & 0.22 \\
\hline
\end{tabular}

Values are means $( \pm \mathrm{SD})$ or numbers of patients (\%).

Continuous variables were compared using the Mann-Whitney $U$ test. The Chi-square test was used for frequency comparisons.

SVD, small vessel disease; VaD, vascular dementia; VaP, vascular parkinsonism; LS, lacunar stroke; BMI, body mass index; CKD, chronic kidney disease; CAD, coronary artery disease; MAP, mean arterial pressure; SBP, systolic blood pressure; DBP, diastolic blood pressure; PAD, peripheral vascular disease; PS, polymetabolic syndrome.

progressed had also increased D-dimer and tended to have low TC and HDL while subjects with VaP tended to have low TG (Table 5).

In the multivariate analysis performed with regard to clinical manifestation of SVD, fibrinogen was associated with risk of any radiological progression in all groups: LS (OR 1.01; 95\% CI (1.002-1.02), $p=0.02$ ), $\mathrm{VaD}$ (OR 1.007; (0.99-1.01), $p=0.07$ ) and VaP (1.002; (1-1.012), $p=0.04$ ). Moreover, HDL (OR 0.97; (0.93-1.001), $p=0.05$ ) and TC (OR 0.98; (0.96-1.001), $p=0.07$ ) were also marginally associated with this risk in $\mathrm{VaD}$ and $\mathrm{TG}$ in VaP (OR 0.98; (0.95-1.002), $p=0.08)$. Area under the ROC curve was comparably large for fibrinogen [AUC 0.65 (0.54-0.76), $p<0.01$ ] and exceeded baseline HDL [AUC $0.55(0.44-0.65)$, $p=0.3$ ] (Fig. 2).

\section{Discussion}

Our study demonstrated on a large group of initially independently living elderly, stratified for 3 different SVD manifestations, that both WMLs and lacunes progressed over time within 2 years of observation. We found that higher concentrations of fibrinogen were associated with an increased risk of radiological progression expressed by new lacunes or WMLs, and our results support the hypothesis that coagulation pathway contributes to the pathogenesis of SVD. This association was independent of age, sex, baseline SVD load and type of SVD manifestations regardless they were subacute (LS) or chronic (VaD or VaP). Interestingly, there were some differences between other associations with the main radiological determinants of SVD. Lower HDL and increased TF predicted development of new lacunes but not WMLs progression while higher BTG was associated with risk of WMLs progression. That difference supports the concept of multiple different mechanisms involved in the pathogenesis and progression of SVD. Lacunar strokes are thought to represent the focal manifestation of actually a diffuse abnormality of the small cerebral arterioles resulting in a state of chronic hypoperfusion of the brain white matter, eventually resulting in degeneration of myelinated fibers as a consequence of repeated selective oligodendrocyte death. Multiple infarcts or WMLs rather denote a more generalized phenomenon such as inflammation, oxidative stress with disruption in the BBB [24].

The study demonstrated that among a panel of different biochemical markers, fibrinogen was the only one that was significantly related to radiological progression in patients with LS, VaD or VaP. These findings suggest haemostatic activation may be an important cause or contributor to progression of SVD. Fibrinogen is an inflammatory marker, and a marker of systemic hypercoagulability acting as an important factor in the coagulation cascade. It has important hemostatic properties as it induces red cell aggregation and affects platelet aggregation and endothelial function. It is also assumed to be a faithful marker of BBB dysfunction. High levels serve as nonspecific marker for inflammatory disease, 
Table 2 - Baseline MRI features of studied patients with and without further radiological progression.

\begin{tabular}{|c|c|c|c|}
\hline Baseline MRI variables & No progression & Radiological progression & $p$ \\
\hline $\mathrm{N}$ & 80 & 43 & - \\
\hline \multicolumn{4}{|l|}{ SVD score } \\
\hline 0 & $2(2.5)$ & 0 & \multirow[t]{5}{*}{0.40} \\
\hline 1 & $23(28.8)$ & $12(27.9)$ & \\
\hline 2 & $22(27.5)$ & 18 (41.9) & \\
\hline 3 & $20(25)$ & $9(20.9)$ & \\
\hline 4 & $13(16.3)$ & $4(9.3)$ & \\
\hline \multicolumn{4}{|l|}{ Fazekas Scale } \\
\hline 1 & $17(21.3)$ & $3(7)$ & \multirow[t]{3}{*}{0.12} \\
\hline 2 & $43(53.8)$ & $28(65.1)$ & \\
\hline 3 & $20(25)$ & $12(27.9)$ & \\
\hline Fazekas Score (mean) & $2(0.76)$ & $2.23(0.53)$ & 0.09 \\
\hline pWMLs & $1.53(0.98)$ & $1.62(0.85)$ & 0.71 \\
\hline dWMLS & $1.47(0.87)$ & $2.04(0.87)$ & $<0.01$ \\
\hline Lacunes & $42(52.5)$ & $18(41.9)$ & 0.34 \\
\hline Microbleeds & 14 (17.5) & 5 (11.6) & 0.44 \\
\hline PVS & $34(42.5)$ & $19(44.2)$ & 0.99 \\
\hline Atrophy & $20(25.3)$ & $13(30.2)$ & 0.67 \\
\hline
\end{tabular}

but also might predispose to thrombosis, reduce blood flow, enhance atherogenesis and lead to increased risk of cerebrovascular diseases. In the ASPS study, higher serum fibrinogen levels were independently associated with WMLs and lacunar lesions on MRI [25]. In patients with symptomatic SVD, fibrinogen has been correlated with the amount of leukoaraiosis in patients with lacunar strokes and $\mathrm{VaD}$ [26]. Also in a systematic review and meta-analysis, concentrations of fibrinogen and D-dimer were higher in chronic lacunar stroke versus non-stroke patients [27]. On the opposite Aribisala et al. found no association between inflammatory biomarkers, fibrinogen and WMLs among 634 community-dwelling older people [28]. We found increased concentrations of BTG especially in patients with WMLs progression what may represent ongoing platelet activation, which is in line with recent study of Yokote et al., who demonstrated elevated release of BTG in patients with atheromatous plaques at brain penetrating arteries [29]. Elevated BTG and other markers of endothelial dysfunction (intercellular adhesion molecule-1 or tissue factor pathway inhibitor) were elevated independent of conventional risk factors in patients with LS and leukoaraiosis [30]. On the other hand patients with new lacunes had increased TF in our study, what may rather reflect prothrombotic changes as TF acts as a trigger for the extrinsic coagulation pathway.

Interestingly, in this study, the concentrations of serum HDL and TC on admission in patients who experienced radiological progression were both lower than those in nonprogressive group and these markers tended to be associated with WMLs or lacunes progression in VaD patients. That finding was in line with the LADIS study results which documented that among 639 elderly subjects with some degree of WMLs on MRI, low HDL was associated with incident lacunes in $\mathrm{VaD}$ subgroup [31]. Other investigators found that mid-life lower HDL concentration was associated with late-life WMLs [32]. The ASPS study also revealed that lower TC was associated with WMLs [33]. In a population based cohorts increasing triglycerides and decreasing LDL were associated with frequency and severity of all MRI markers of SVD in older community persons [11]. However, findings on the association between cholesterol fractions levels and SVD are not consistent. LDL, total cholesterol and triglicerydes were not independent risk factors for any SVD subgroups in LADIS and in the cross-sectional ARIC study of 1827 communitydwelling participants, incident lacunes related to lipohyalinosis were associated with LDL but not TC [34]. Also in a Rotterdam Scan Study progression of SVD was related to vascular risk factors (age, female sex, smoking, hypertension), baseline lesion load but not TC and HDL [35]. On the opposite, another recent study documented that lower TC and HDL levels were associated with the presence of another SVD radiological marker - deep CMBs [36].

The relationship between plasma or imaging markers and radiological progression of SVD is not clear. Although SVD is considered to result from cerebral arteriolar occlusive disease, the pathogenesis is largely unknown and probably the exact mechanisms of SVD radiological and clinical manifestations differ [37]. Classical cardiovascular risk factors are not consistently common in patients with SVD and pathophysiology of SVD may be independent from that of atherosclerotic large artery disease and other, nonvascular contributing mechanisms play a role in the cause of SVD [38]. Probably the influence of systemic factors on the endothelium results in increase in permeability of the BBB what initiates pathologic changes. In majority of the studies, the causal relationship between biochemical markers cannot be determined because majority of them had cross-sectional design. We cannot directly compare our results with others because studies on SVD radiological progression usually concentrated on inflammatory biomarkers and did not focus on hemostatic factors. It is of interest that, we observed some difference in biochemical profiles between LS, VaD and VaP. Although this observation requires replication to ensure validity, if validated, it lends support to the involvement of different pathways in the 
Table 3 - Comparison of biochemical markers of studied patients without and with any radiological progression, with new lacunes or with WMLs progression.

\begin{tabular}{|c|c|c|c|c|c|c|c|}
\hline \multirow[t]{2}{*}{ Variables } & \multicolumn{3}{|c|}{ New lacunes or WMLs progression } & \multicolumn{2}{|c|}{ New lacunes } & \multicolumn{2}{|c|}{ WMLs progression } \\
\hline & No & Yes & $p^{*}$ & Yes & $p^{*}$ & Yes & $p^{*}$ \\
\hline$N$ & 80 & 43 & - & 25 & - & 33 & - \\
\hline Age (years) & $71.25(8.07)$ & $73.8(8)$ & 0.1 & $72.90(8.8)$ & 0.55 & $72.78(7)$ & 0.46 \\
\hline MPV (fl) & $9.22(1.1)$ & $9.27(2)$ & 0.84 & $9.1(1.2)$ & 0.75 & $9.10(2.69)$ & 0.78 \\
\hline hsCRP (mg/dl) & $0.68(1.9)$ & $0.85(1.2$ & 0.6 & $0.67(1.01)$ & 0.98 & $0.51(0.9)$ & 0.72 \\
\hline PGL (mg/dl) & $121.8(43.5)$ & $123.53(49.8)$ & 0.84 & $135.7(70)$ & 0.38 & $113.61(38)$ & 0.46 \\
\hline HBA1c (\%) & $6.398(1.1)$ & $6.270(0.92)$ & 0.53 & $6.26(0.76)$ & 0.75 & $6.34(1.1)$ & 0.85 \\
\hline eGFR $\left(\mathrm{ml} / \mathrm{min} / 1.73 \mathrm{~m}^{2}\right)$ & $81.17(23.1)$ & $72.45(26)$ & 0.06 & $66.2(35.1)$ & 0.07 & $68.03(21.3)$ & 0.03 \\
\hline LDL-C (mg/dl) & $112.29(39.4)$ & $102.8(33.6)$ & 0.18 & $110.3(37.6)$ & 0.88 & $102.52(30.7)$ & 0.33 \\
\hline HDL-C (mg/dl) & $51.43(15)$ & $45.8(14.9)$ & 0.05 & $39.1(16.5)$ & 0.01 & $47.73(14.4)$ & 0.35 \\
\hline TG (mg/dl) & $138.06(90.7)$ & $112.36(54.7)$ & 0.09 & $114.3(41.7)$ & 0.42 & $122.87(70.4)$ & 0.51 \\
\hline TC (mg/dl) & $189.46(48)$ & $171.2(34.2)$ & 0.03 & $174.8(25.6)$ & 0.35 & $172.03(31.4)$ & 0.15 \\
\hline D-dimer $(\mu \mathrm{g} / \mathrm{ml})$ & $0.72(0.6)$ & $1.14(1.2)$ & 0.03 & $0.86(0.7)$ & 0.59 & $0.86(1.04)$ & 0.50 \\
\hline Fibrinogen (mg/dl) & $329.74(85)$ & $382.33(87.2)$ & 0.003 & $384.56(116.6)$ & 0.08 & $382.75(92.8)$ & 0.03 \\
\hline BTG (ng/ml) & $25.27(43.1)$ & 47.09 (92.4) & 0.13 & $45.79(7.1)$ & 0.28 & $55.06(90.08)$ & 0.06 \\
\hline sTM (ng/ml) & $5.7(4.4)$ & $5.4(1.7)$ & 0.92 & $6.0(2.7)$ & 0.82 & $5.9(2.1)$ & 0.85 \\
\hline $\mathrm{TF}(\mathrm{pg} / \mathrm{ml})$ & $49.77(15.4)$ & $61.9(22.1)$ & 0.04 & $80.84(17.6)$ & 0.003 & 38.7 (14.8) & 0.33 \\
\hline
\end{tabular}

Values are means $( \pm \mathrm{SD})$.

* Compared with patients with no radiological progression.

WMLs, white matter lesions; sTM, soluble thrombomodulin; TF, tissue factor; MPV, mean platelet volume; PGL, plasma glucose level; BTG, beta thromboglobulin; TF, tissue factor.

Table 4 - Multivariate analysis of independent variables associated with SVD progression.

\begin{tabular}{lcc} 
Variables & New lacunes or WMLs progression & $p$ \\
\hline Fibrinogen & $1.02(1.006-1.011)$ & 0.01 \\
HDL & $0.97(0.94-1.005)$ & 0.04 \\
TC & $0.99(0.97-1.001)$ & 0.09 \\
Variables & New lacunes & $p$ \\
\hline Fibrinogen & $1.02(1.003-1.05)$ & 0.02 \\
HDL & $0.96(0.93-1.0)$ & 0.05 \\
TF & $1.07(0.99-1.1)$ & 0.07 \\
Variables & WMLs progression & $p$ \\
\hline Fibrinogen & $1.007(1.001-1.01)$ & 0.01 \\
BTG & $1.005(0.99-1.01)$ & 0.07 \\
\hline
\end{tabular}

Values are OR (95\% CI); WMLs, white matter lesions.

TF, tissue factor; BTG, beta thromboglobulin; TC, total cholesterol; HDL, high density lipoprotein.

pathogenesis of LS, VaD or VaP. Little is known about natural radiological course of $\mathrm{VaP}$ and $\mathrm{VaD}$ caused by SVD, which makes our data the more important. The present study documented that patients with LS and VaP had similar risk for radiological progression, however patients with $\mathrm{VaD}$ tended to have this risk higher. Majority of our patients with SVD had moderate or severe WMLs on baseline. Several longitudinal studies have investigated the rate and predictors for progression of WMLs, and the most consistent predictor for progression of WMLs is the baseline severity of WMLs. Patients with punctuate WMLs usually have minimal progression of WMLs, whereas those with early confluent and confluent WMLs at baseline have rapid progression of WMLs. While many studies report an association between atrophy and SVD it is not specific to SVD and it may occur in normal aging.
We acknowledge that there are several limitations of this study. The major weakness is the small number of patients from a single center with data-driven approaches to selecting variables what might have produced biased estimates and the results may not be generalizable to other population. The number of patients in each group was small which decreased the power of our study in detecting some significant differences among different groups. However, this is also a limiting factor in most published reports on the subject. Second, because of its single-center observational nature, selection and severity bias may be reflected in the characteristics of our study population. It is also possible that certain unobserved factors such as genetic background and pathophysiology of SVD may also influence the likelihood of SVD progression after controlling most associated risk factors. The study is therefore regarded as hypothesis generating rather than definitive, and a larger study and replication are needed for more robust conclusions. Although patients with $\mathrm{VaP}$ and $\mathrm{VaD}$ were included to the present study immediately after diagnosis but they were in an advanced stage of their disease, therefore it remains unknown whether our results can be applied to less severely affected patients. Measuring progression of WMLs burden is yet a further challenge and the major weakness was a visual assessment of the imaging. However, Fazekas scale remains the most widely used system for describing white matter disease severity in publications and it is histopathologically related with SVD [39]. Fully quantitative techniques also suffer from several pitfalls, including changes in signal intensity of the WMLs or aging brain [40]. These problems usually aggravates in a multicenter setting with the use of different MRI scanners which cannot provide completely identical image sequences. The overall study period in our study (24 months) could ideally have been longer to reach more significant results. Therefore, future studies are needed to develop a predictive risk score for chronic manifestations of 
Table 5 - Comparison of baseline biochemical markers of studied patients with lacunar stroke, vascular dementia and parkinsonism without and with any radiological progression.

\begin{tabular}{|c|c|c|c|c|c|c|c|c|c|}
\hline \multirow[t]{2}{*}{ Variables } & \multicolumn{3}{|c|}{ Lacunar stroke } & \multicolumn{3}{|c|}{$\mathrm{VaD}$} & \multicolumn{3}{|c|}{ VaP } \\
\hline & No progression & Progression & $p$ & No progression & Progression & $p$ & No progression & Progression & $p$ \\
\hline$N$ & 36 & 13 & - & 27 & 21 & - & 17 & 9 & - \\
\hline Age (years) & $69.72(9.3)$ & $69.46(7)$ & 0.93 & $72.93(6.9)$ & $75.86(9)$ & 0.21 & $71.82(6.5)$ & $75.33(4)$ & 0.16 \\
\hline Hypertension & 33 (91.7) & $13(100)$ & 0.55 & $24(88.9)$ & $18(85.7)$ & 0.9 & $12(70.6)$ & $6(66.7)$ & 0.9 \\
\hline CAD & $9(25)$ & $1(7.7)$ & 0.25 & $8(29.6)$ & $4(19)$ & 0.51 & $1(5.9)$ & $1(11.1)$ & 0.9 \\
\hline Diabetes mellitus & $21(58.3)$ & $7(53.6)$ & 0.9 & $15(55.6)$ & $12(57.1)$ & 0.9 & $9(52.9)$ & $4(44.4)$ & 0.9 \\
\hline Current smoking & $12(33.3)$ & $4(30.8)$ & 0.9 & $6(22.2)$ & $4(19)$ & 0.9 & 7 (41.2) & $4(44.4)$ & 0.9 \\
\hline Hyperlipidemia & $27(75)$ & $10(76.9)$ & 0.9 & $23(85.2)$ & $13(61.9)$ & 0.09 & $13(76.5)$ & $5(55.6)$ & 0.38 \\
\hline PAD & $1(2.8)$ & 0 & 0.9 & $2(7.4)$ & $2(9.5)$ & 0.9 & $2(11.8)$ & $3(33.3)$ & 0.3 \\
\hline PS & $18(50)$ & $6(46.2)$ & 0.9 & $13(48.1)$ & $8(38.1)$ & 0.56 & $6(35.3)$ & $2(22.2)$ & 0.66 \\
\hline Obesity (BMI $\geq 30 \mathrm{~kg} / \mathrm{m}^{2}$ ) & $9(25)$ & $7(53.8)$ & 0.08 & $6(22.2)$ & $5(23.8)$ & 0.9 & $2(11.8)$ & $2(22.2)$ & 0.59 \\
\hline CKD & $4(11.1)$ & $5(38.5)$ & 0.04 & $2(7.4)$ & $3(14.3)$ & 0.64 & $2(11.8)$ & $2(22.2)$ & 0.59 \\
\hline Use of statin medications & $33(91.7)$ & $12(92.3)$ & 0.99 & $22(81.5)$ & $13(61.9)$ & 0.19 & $9(52.9)$ & 7 (77.8) & 0.39 \\
\hline Antihypertensive therapy & $33(91.7)$ & $11(84)$ & 0.72 & $22(81)$ & $18(85.7)$ & 0.75 & $12(70.6)$ & $6(66.7)$ & 0.9 \\
\hline Daily use of aspirin & $35(97.2)$ & $13(100)$ & 0.99 & $21(77.8)$ & $14(66.7)$ & 0.51 & $12(70.6)$ & $5(55.6)$ & 0.66 \\
\hline hsCRP (mg/dl) & $0.88(2.7)$ & $0.50(0.8)$ & 0.63 & $0.40(0.6)$ & $1.04(1.4)$ & 0.05 & $0.69(0.9)$ & $0.95(1.2)$ & 0.57 \\
\hline D-dimer $(\mu \mathrm{g} / \mathrm{ml})$ & $0.7(0.8)$ & $0.60(0.2)$ & 0.48 & $0.64(0.5)$ & $1.42(1.4)$ & 0.04 & $0.68(0.4)$ & $1.33(1.5)$ & 0.19 \\
\hline Fibrinogen (mg/dl) & $331.4(72)$ & 385 (71.1) & 0.04 & $325.9(92.2)$ & $389.0(92.3)$ & 0.04 & $331.0(105.8)$ & $361.8(101.3)$ & 0.04 \\
\hline MPV (fl) & $9.1(1.1)$ & $9.28(1.7)$ & 0.83 & $9.0(1)$ & $8.94(2.2)$ & 0.91 & $9.56(0.88)$ & $10.06(1.7)$ & 0.35 \\
\hline LDL-C (mg/dl) & $109.6(42.1)$ & $94.6(31.5)$ & 0.25 & $117.4(37.7)$ & $105.9(35.4)$ & 0.30 & $110.2(37.3)$ & $107.3(33.8)$ & 0.85 \\
\hline HDL (mg/dl) & $47.4(10.3)$ & $44.3(9.1)$ & 0.35 & $53.75(20.5)$ & $43.57(16.5)$ & 0.08 & $56.51(12.3)$ & 53.13 (16.7) & 0.57 \\
\hline TG (mg/dl) & $150(118)$ & $131.6(76.4)$ & 0.61 & $116.96(51.8)$ & $106.6(41.9)$ & 0.47 & $142.8(58.4)$ & $97.9(40.8)$ & 0.05 \\
\hline TC (mg/dl) & $179.5(47.4)$ & $163.7(28.8)$ & 0.27 & $199.42(50.1)$ & $173(33.5)$ & 0.05 & $196.2(44.2)$ & 177.9 (43.9) & 0.33 \\
\hline PGL (mg/dl) & $126.8(46.9)$ & $148.6(66)$ & 0.21 & $115(42.1)$ & $117.5(42.6)$ & 0.84 & $121.38(38.6)$ & $101.3(17.1)$ & 0.16 \\
\hline eGFR $\left(\mathrm{ml} / \mathrm{min} / 1.73 \mathrm{~m}^{2}\right)$ & $81.13(29.1)$ & $74.1(26.5)$ & 0.45 & $84.74(15.7)$ & $74.9(26.2)$ & 0.14 & $76.13(15.6)$ & $64.28(26.4)$ & 0.17 \\
\hline $\mathrm{BTG}(\mathrm{ng} / \mathrm{ml})$ & $21.2(36.3)$ & $59.5(102)$ & 0.14 & $23.33(35)$ & $54.79(102.2)$ & 0.19 & $34.04(62)$ & $5.03(7.9)$ & 0.28 \\
\hline sTM (ng/ml) & $6.2(6.5)$ & $6.5(2.2)$ & 0.90 & $5.2(1.1)$ & $5.4(2.0)$ & 0.74 & $5.3(5.9)$ & $5.7(2.0)$ & 0.47 \\
\hline $\mathrm{TF}(\mathrm{pg} / \mathrm{ml})$ & $49.88(11)$ & $66.2(21.6)$ & 0.40 & $53.72(23.3)$ & $62.5(23.9)$ & 0.44 & $38.59(14.8)$ & $58.88(9.6)$ & 0.19 \\
\hline
\end{tabular}

Values are means $( \pm \mathrm{SD})$ or numbers of patients (\%).

VaD, vascular dementia; VaP, vascular parkinsonism; LS, lacunar stroke; sTM, soluble thrombomodulin; TF, tissue factor; MPV, mean platelet volume; PGL, plasma glucose level; BTG, beta thromboglobulin; TF, tissue factor; CAD, coronary artery disease; PAD, peripheral vascular disease; PS, polymetabolic syndrome.

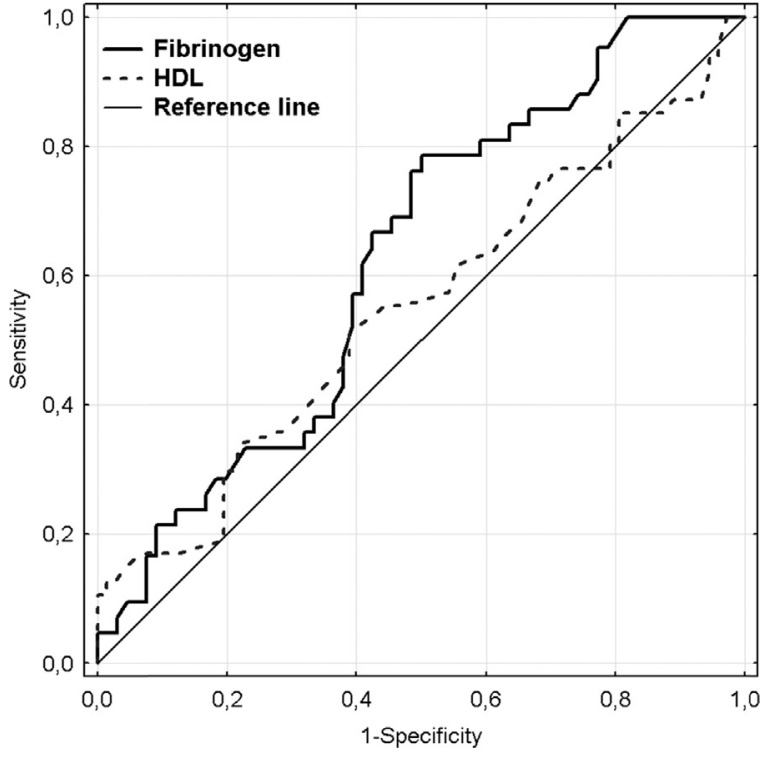

Fig. 2 - ROC curves for baseline fibrinogen and HDL.

SVD, and to clarify the interaction between degenerative and vascular processes in their development. On the other hand our study has some advantages. We included a well- phenotyped group of patients with SVD including rarely studied chronic VaP and VaD subjects using uniform diagnostic procedures. Second, our study had 24-months of follow-up with repeated MRI examinations, what allowed us to assess the long-term effects of different baseline measures on radiological progression.

\subsection{Summary}

In conclusion, our results support that hemostatic factors and lipid metabolism play a role in SVD radiological progression. Fibrinogen concentration was independently associated with an increased risk of radiological progression expressed by new lacunes or WMLs progression regardless of the clinical SVD manifestation. In addition, the two radiological subtypes of SVD demonstrated differences in other associated biochemical markers. Lower HDL and increased TF predicted development of new lacunes, and higher BTG was associated with risk of WMLs progression. Further research is required to ensure the validity of our findings.

\section{Conflict of interest}

None declared. 


\section{Acknowledgment and financial support}

This study was supported by the Polish Ministry of Science and Higher Education as a research project of the Military Institute of Medicine (Warsaw, Poland, study number N N402 473840).

\section{REFERENCES}

[1] Pantoni L. Cerebral small vessel disease: from pathogenesis and clinical characteristics to therapeutic challenges. Lancet Neurol 2010;9:689-701.

[2] Wardlaw J, Smith C, Dichgans M. Mechanisms underlying sporadic cerebral small vessel disease: insights from neuroimaging. Lancet Neurol 2013;12:70060-7.

[3] Schmidt R, Schmidt H, Haybaeck J, Loitfelder M, Weis S, Cavalieri $\mathrm{M}$, et al. Heterogeneity in age-related white matter changes. Acta Neuropathol 2011;122:171-85.

[4] Gouw AA, van der Flier WM, Fazekas F, van Straaten EC, Pantoni L, Poggesi A, et al. Progression of white matter hyperintensities and incidence of new lacunes over a 3-year period: the Leukoaraiosis and Disability study. Stroke 2008;39:1414-20.

[5] Jackson C, Sudlow C. Are lacunar strokes really different? A systematic review of differences in risk factor profiles between lacunar and nonlacunar infarcts. Stroke 2005;36:891-901.

[6] Liao D, Cooper I, Cai J, Toole J, Bryan N, Burke G, et al. The prevalence and severity of white matter lesions, their relationship with age, ethnicity, gender, and cardiovascular risk factors: the ARIC study. Neuroepidemiology 1997;16:149-62.

[7] Longerstreth Jr WT, Arnold AM, Beauchamp Jr NJ, Manolio TA, Lefkowitz D, Jungreis C, et al. Incidence, manifestations, and predictors of worsening white matter on serial cranial magnetic resonance imaging in the elderly: the Cardiovascular Health Study. Stroke 2005;36:56-61.

[8] Norden AGW, Laat KF, Gons RAR. Causes and consequences of cerebral small vessel disease. The RUN DMC study: a prospective cohort study. Study rationale and protocol. BMC Neurol 2011;11:29.

[9] Wiseman SJ, Doubal FN, Chappell FM, Valdés-Hernández MC, Wang XI, Rumley A, et al. Plasma biomarkers of inflammation, endothelial function and hemostasis in cerebral small vessel disease. Cerebrovasc Dis 2015;40:15764.

[10] Grotemeyer KC, Kaiser R, Grotemeyer KH, Husstedt IW. Association of elevated plasma viscosity with small vessel occlusion in ischemic cerebral disease. Thromb Res 2014;133:96-100.

[11] Schilling S, Tzourio C, Dufouil C, Zhu Y, Berr C, Alpérovitch A, et al. Plasma lipids and cerebral small vessel disease. Neurology 2014;11:1844-52.

[12] Staszewski J, Piusińska-Macoch R, Skrobowska E. Significance of haemodynamic and haemostatic factors in the course of different manifestations of cerebral small vessel disease: the SHEF-SVD Study-Study Rationale and Protocol. Neurosci J 2013;9 pages. Article ID 424695.

[13] Hurtig HI. Vascular parkinsonism. In: Stern MB, Koller WC, editors. Parkinsonian syndromes. New York: Marcel Dekker; 1993. p. 81-3.

[14] Chui HC, Victoroff JI, Margolin D. Criteria for the diagnosis of ischemic vascular dementia proposed by the State of California Alzheimer's Disease Diagnostic and Treatment Centers. Neurology 1992;42:473-80.
[15] Zijlmans JCM, Daniel SE, Hughes AJ. Clinicopathological investigation of vascular parkinsonism, including clinical criteria for diagnosis. Mov Disord 2004;19:630-40.

[16] Wardlaw JM, Smith EE, Biessels GJ, Cordonnier C, Fazekas F, Frayne R, et al. Neuroimaging standards for research into small vessel disease and its contribution to ageing and neurodegeneration. Lancet Neurol 2013;12:822-38.

[17] Staals J, Makin SDJ, Doubal FN, Dennis MS, Wardlaw JM. Stroke subtype, vascular risk factors, and total MRI brain small-vessel disease burden. Neurology 2014;30:1228-34.

[18] Fazekas F, Chawluk JB, Alavi A. MR signal abnormalities at $1.5 \mathrm{~T}$ in Alzheimer's dementia and normal aging. AJR Am J Roentgenol 1987;149:351-6.

[19] Pantoni L, Basile AM, Pracucci G, Asplund K, Bogousslavsky $\mathrm{J}$, Chabriat $\mathrm{H}$, et al. Impact of age-related cerebral white matter changes on the transition to disability: the LADIS study: rationale, design and methodology. Neuroepidemiology 2005;24:51-62.

[20] Valdes Hernandez Mdel C, Morris Z, Dickie DA, Royle NA, Muñoz Maniega S, Aribisala BS, et al. Close correlation between quantitative and qualitative assessments of white matter lesions. Neuroepidemiology 2013;40:13-22.

[21] Doubal FN, MacLullich AM, Ferguson KJ, Dennis MS, Wardlaw JM. Enlarged perivascular spaces on MRI are a feature of cerebral small vessel disease. Stroke 2010;41:450-4.

[22] Prins ND, van Straaten EC, van Dijk EJ, Simoni M, van Schijndel RA, Vrooman HA, et al. Measuring progression of cerebral white matter lesions on MRI: visual rating and volumetrics. Neurology 2004;62:1533-9.

[23] Staszewski J, Piusińska-Macoch R, Skrobowska E, Brodacki B, Macek K, Stępień A. Vascular parkinsonism and vascular dementia are associated with an increased risk of vascular events or death. Arch Med Sci Atheroscler Dis 2017;2(1):e1623. http://dx.doi.org/10.5114/amsad.2017.68549

[24] Wardlaw JM. What causes lacunar stroke? J Neur Neurosurg Psychiatry 2005;76:617-9.

[25] Schmidt R, Fazekas F, Hayn M, Schmidt H, Kapeller P, Roob $G$, et al. Risk factors for microangiopathy-related cerebral damage in the Austrian Stroke Prevention Study. J Neurol Sci 1997;152:15-21.

[26] Marti-Fabregas J, Valencia C, Pujol J, Garcia-Sanchez C, Marti-Vilalta JL. Fibrinogen and the amount of leukoaraiosis in patients with symptomatic small-vessel disease. Eur Neurol 2002;48:185-90.

[27] Wiseman S, Marlborough F, Doubal F, Webb DJ, Wardlaw J. Blood markers of coagulation, fibrinolysis, endothelial dysfunction and inflammation in lacunar stroke versus non-lacunar stroke and non-stroke: systematic review and meta-analysis. Cerebrovasc Dis 2014;37:64-75.

[28] Aribisala BS, Wiseman S, Morris Z, Valdés-Hernández MC, Royle NA, Maniega SM, et al. Circulating inflammatory markers are associated with magnetic resonance imagingvisible perivascular spaces but not directly with white matter hyperintensities. Stroke 2014;45:605-7.

[29] Yokote A, Hashimoto K, Bikei R, Nakamoto H. Elevated release of beta-thromboglobulin and platelet factor 4 in cerebral infarction patients with branch atheromatous disease: a preliminary report. Neurol Med Chir 2015;55:809-12.

[30] Hassan A, Hunt BJ, O'Sullivan M, Parmar K, Bamford JM, Briley D, et al. Markers of endothelial dysfunction in lacunar infarction and ischaemic leukoaraiosis. Brain 2003;126:424-32.

[31] Gouw AA, van der Flier WM, Fazekas F, van Straaten EC, Pantoni L, Poggesi A, et al. Progression of white matter hyperintensities and incidence of new lacunes over a threeyear period: the Leukoaraiosis and Disability study. Stroke 2008;39:1414-20. 
[32] Carmelli D, Swan GE, Reed T, Wolf PA, Miller BL, DeCarli C. Midlife cardiovascular risk factors and brain morphology in identical older male twins. Neurology 1999;52:1119-24.

[33] Schmidt R, Hayn M, Fazekas F, Kapeller P, Esterbauer H. Magnetic resonance imaging white matter hyperintensities in clinically normal elderly individuals. Correlations with plasma concentrations of naturally occurring antioxidants. Stroke 1996;27:2043-7.

[34] Bezerra DC, Sharrett AR, Matsushita K, Gottesman RF, Shibata D, Mosley Jr TH, et al. Risk factors for lacune subtypes in the atherosclerosis risk in communities (ARIC) study. Neurology 2012;78:102-8.

[35] van Dijk EJ, Prins ND, Vrooman HA, Hofman A, Koudstaal PJ, Breteler MM. Progression of cerebral small vessel disease in relation to risk factors and cognitive consequences: Rotterdam Scan study. Stroke 2008;39:2712-9.
[36] Mitaki S, Nagai A, Oguro H, Yamaguchi S. Serum lipid fractions and cerebral microbleeds in a healthy Japanese population. Cerebrovasc Dis 2017;43:186-91.

[37] Wardlaw JM, Smith C, Dichgans M. Mechanisms underlying sporadic cerebral small vessel disease: insights from neuroimaging. Lancet Neurol 2013;12:532.

[38] Mok V, Gorelick PB, Chen C. Risk factors as possible targets for prevention of small vessel disease. In: Pantoni L, Gorelic PB, editors. Cerebral small vessel disease, 2014.

[39] Fazekas F, Kleinert R, Offenbacher H, Schmidt R, Kleinert G, Payer F, et al. Pathologic correlates of incidental MRI white matter signal hyperintensities. Neurology 1993;43:1683-9.

[40] Olsson E, Klasson N, Berge J, Eckerström C, Edman A, Malmgren $\mathrm{H}$, et al. White matter lesion assessment in patients with cognitive impairment and healthy controls: reliability comparisons between visual rating, a manual and an automatic volumetrical MRI method - the Gothenburg MCI study. J Aging Res 2013. Article ID 198471. 